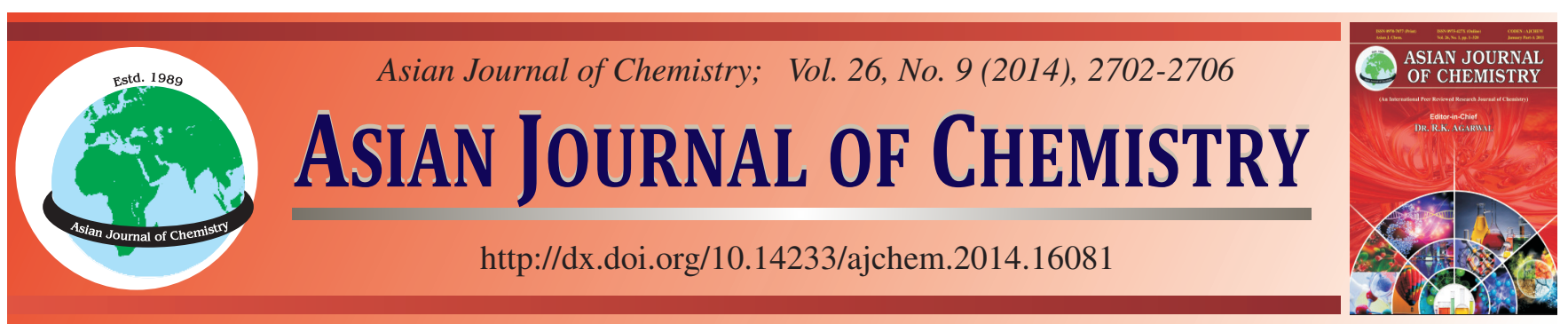

\title{
Kinetics and Mechanism of Oxidation of Aliphatic Secondary Alcohols by Benzyltrimethylammonium Chlorobromate
}

\author{
PradeEP K. Sharma
}

Department of Chemistry, J.N.V. University, Jodhpur-342 005, India

Corresponding author: E-mail: drpkvs27@yahoo.com

\begin{abstract}
Oxidation of several secondary alcohols by benzyltrimethylammonium chlorobromate (BTMACB) in aqueous acetic acid leads to the formation of corresponding ketones. The reaction is first order with respect to BTMACB and the alcohols. The reaction failed to induce the polymerization of acrylonitrile. There is no effect of tetrabutylammonium chloride on the reaction rate. The proposed reactive oxidizing species is chlorobromate ion. The oxidation of benzhydrol- $\alpha-\mathrm{d}(\mathrm{PhCDOHPh})$ exhibited a substantial primary kinetic isotope effect $\left(\mathrm{k}_{\mathrm{H}} / \mathrm{k}_{\mathrm{D}}\right.$ $=5.61$ at $298 \mathrm{~K}$ ). The effect of solvent composition indicated that the rate increases with an increase in the polarity of the solvent. The reaction is susceptible to both the polar and steric effects of the substituents. A mechanism involving transfer of a hydride ion in the ratedetermining step has been proposed.
\end{abstract}

Keywords: Alcohols, Correlation analysis, Chlorobromate, Kinetics, Mechanism, Oxidation.

\section{INTRODUCTION}

Benzyltrimethylammonium chlorobromate [BTMACB or $\left.\left(\mathrm{PhCH}_{2} \mathrm{NMe}_{3}\right)^{+}\left(\mathrm{Br}_{2} \mathrm{Cl}\right)^{-}\right]$is one of the quaternary ammonium polyhalides which has been used as an effective halogenating and oxidizing agent in synthetic organic chemistry ${ }^{1-3}$. Polymeric benzyltrimethylammonium dichloroiodate and dibromoiodate have been used for the addition of halogens to olefins ${ }^{4}$. The polyhalides are more suitable than molecular halogens because of their solid nature, ease of handling, stability, selectivity and excellent product yield. We have been interested in the kinetic and mechanistic aspects of oxidation by polyhalogen compounds. A few reports (including BTMACB) regarding the kinetic and mechanistic studies of the reactions of polyhalides are available in the literature ${ }^{5-8}$. There seems to be no report on the oxidation of aliphatic secondary alcohols by BTMACB. Therefore, in continuation of our earlier work on the oxidation studies by polyhalides ${ }^{9-11}$, We report here the kinetics of the oxidation of eight aliphatic secondary alcohols, 1-phenylethanol and benzhydrol by BTMACB in aqueous acetic acid solution. Attempts have been made to correlate rate and structure in this reaction. A suitable mechanism has also been proposed.

\section{EXPERIMENTAL}

All the alcohols were commercial products (Fluka) and were dried over magnesium sulphate and than fractionated.
Benzhydrol was recrystallized from ethanol. Benzyltrimethylammonium chlorobromate was prepared by reported method ${ }^{1}$ and its purity was checked by iodometric method. Benzhydrol$\alpha$-d (PhCDOHPh) was also prepared by reported method ${ }^{12}$. The isotopic purity of $\mathrm{PhCDOPh}$, as ascertained by its NMR spectra, was $93 \pm 5 \%$. Acetic acid was refluxed with chromic oxide and acetic anhydride for $6 \mathrm{~h}$ and then it was fractionally distilled. All other reagents were commercial products and were purified by the usual methods ${ }^{13}$.

Product analysis: The product analysis was carried out under kinetic conditions i.e. with an excess of alcohol over BTMACB. In a typical experiment, 2-propanol $(0.05 \mathrm{~mol})$, $\operatorname{KBr}(0.2 \mathrm{~mol})$ and BTMACB $(0.01 \mathrm{~mol})$ were made up to 100 $\mathrm{mL}$ in $1: 1(\mathrm{v} / \mathrm{v})$ aqueous acetic acid and kept in the dark for ca. $10 \mathrm{~h}$ to ensure completion of the reaction. The solution was then treated with an excess $(200 \mathrm{~mL})$ of a saturated solution of 2,4-dinitrophenylhydrazine in $2 \mathrm{~mol} \mathrm{dm}^{-3} \mathrm{HCl}$ and kept overnight in a refrigerator.

The precipitated 2,4-dinitrophenyl-hydrazone (DNP) was filtered off, dried, weighed, recrystallized from ethanol and weighed again. The yields of DNP before and after recrystallization were $2.21 \mathrm{~g}(93 \%)$ and $1.97 \mathrm{~g}(81 \%)$, respectively, based on the consumption of BTMACB. The DNP was found to be identical (m.p. and mixed m.p.) with the DNP of acetone. In similar experiments, with other alcohols, the yields of the DNP were in the range of 78-89\% after recrystallization. 
Kinetic measurements: The reactions were studied under pseudo-first-order conditions by keeping an excess $(\times 15$ or more) of the substrate over BTMACB. The solvent was 1:1(v/ v) acetic acid-water, unless mentioned otherwise. The reactions were studied at constant temperature $( \pm 0.1 \mathrm{~K})$ and were followed by monitoring the decrease in the concentration of BTMACB at $394 \mathrm{~nm}$ for up to $80 \%$ reaction. Pseudo-first-order rate constants, $\mathrm{k}_{\mathrm{obs}}$, were evaluated from linear plots $(\mathrm{r}>0.995)$ of $\log$ [BTMACB] against time. Duplicate kinetic runs showed that the rate constants are reproducible to within $\pm 3 \%$. Simple and multivariate regression analyses were carried out by the least-squares method.

\section{RESULTS AND DISCUSSION}

The rate constants and other experimental data were obtained for all the alcohols. Since the results are similar, only representative data are reproduced here.

The oxidation of alcohols by BTMACB results in the formation of corresponding ketones. The overall reaction may be represented as equation (1).

$$
\mathrm{R}_{2} \mathrm{CHOH}+\mathrm{Br}_{2} \mathrm{Cl}^{-} \longrightarrow \mathrm{R}_{2} \mathrm{CO}+2 \mathrm{H}^{+}+2 \mathrm{Br}^{-}+\mathrm{Cl}^{-} \text {(1) }
$$

Rate laws: The reactions are of first order with respect to BTMACB. Further, the pseudo-first order rate constants, $\mathrm{k}_{\mathrm{obs}}$ are independent of the initial concentration of BTMACB. The rate constant increases linearly with an increase in the concentration of the alcohol (Table-1). Fig. 1 depicts a typical kinetic run.

\begin{tabular}{cccc}
\multicolumn{5}{c}{ TABLE-1 } \\
\multicolumn{4}{c}{$\begin{array}{c}\text { RATE CONSTANTS FOR THE OXIDATION OF } \\
\text { 2-PROPANOL BY BTMACB AT 298 K }\end{array}$} \\
\hline $\begin{array}{c}10^{3}[\mathrm{BTMACB}] \\
\left(\mathrm{mol} \mathrm{dm}^{-3}\right)\end{array}$ & $\begin{array}{c}{[\text { Alcohol] }} \\
\left(\mathrm{mol} \mathrm{dm}^{-3}\right)\end{array}$ & $\begin{array}{c}10^{4} \mathrm{k}_{\mathrm{obs}} \\
\left(\mathrm{s}^{-1}\right)\end{array}$ & $\begin{array}{c}10^{3} \mathrm{k}_{2} \\
\left(\mathrm{dm}^{3} \mathrm{~mol}^{-1} \mathrm{~s}^{-1}\right)\end{array}$ \\
\hline 1.0 & 0.10 & 3.58 & 3.58 \\
1.0 & 0.20 & 7.10 & 3.55 \\
1.0 & 0.40 & 14.4 & 3.60 \\
1.0 & 0.60 & 21.3 & 3.55 \\
1.0 & 0.80 & 28.2 & 3.53 \\
1.0 & 1.00 & 35.1 & 3.51 \\
1.0 & 1.50 & 53.4 & 3.56 \\
2.0 & 0.20 & 7.15 & 3.75 \\
4.0 & 0.20 & 6.75 & 3.38 \\
6.0 & 0.20 & 7.29 & 3.65 \\
8.0 & 0.20 & 6.93 & 3.47 \\
1.0 & 0.40 & 15.3 & 3.83 \\
\hline${ }^{*}$ contained $0.005 \mathrm{~mol} \mathrm{dm}^{-3}$ acrylonitrile &
\end{tabular}

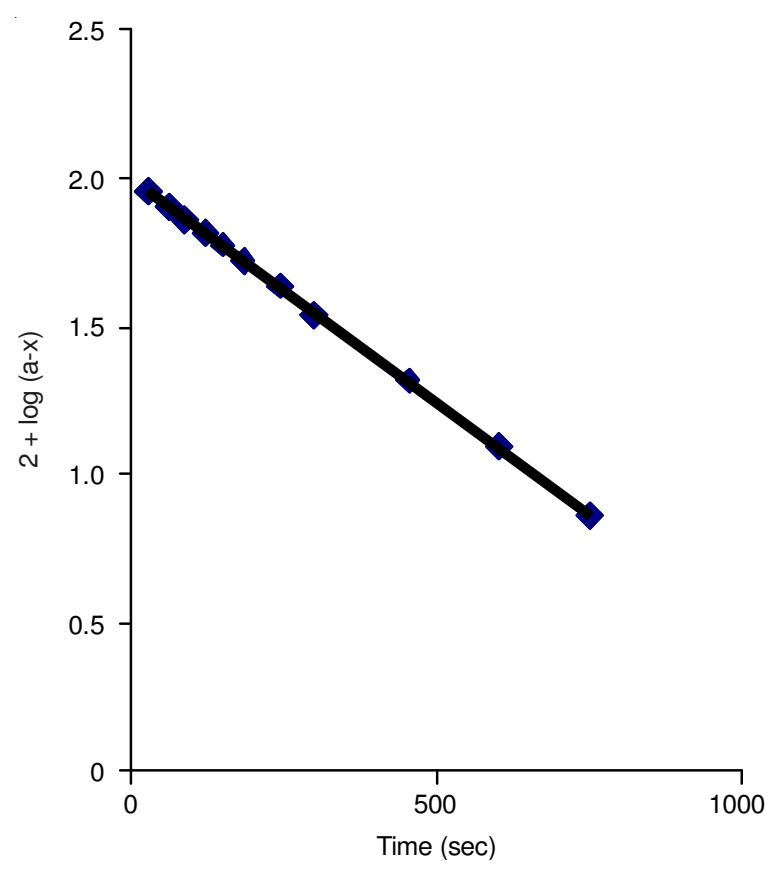

Fig. 1. Oxidation of 2-propanol by BTMACB: A typical kinetic run

Induced polymerization of acrylonitrile: The oxidation of 2-propanol, in an atmosphere of nitrogen, failed to induce the polymerization of acrylonitrile. Further, the addition of acrylonitrile had no effect on the rate of oxidation (Table-1).

Kinetic isotope effect: To ascertain importance of the cleavage of the $\alpha-\mathrm{C}-\mathrm{H}$ bond in the rate-determining step, the oxidation of benzhydrol- $\alpha-\mathrm{d}(\mathrm{PhCDOHPh})$ was studied. Results showed the presence of a substantial primary kinetic isotope effect (Table-2).

Effect of tetrabutylammonium chloride: Addition of tetrabutylammonium chloride (TBACl) had no effect on the rates of oxidation (Table-3).

Effect of solvent composition: The rate constant of oxidation was determined in solvents containing different amounts of acetic acid and water. It was observed that the rate constant increased with an increase in the amount of water in the solvent mixture (Table-4).

Effect of temperature: The rate constant of oxidation of ten secondary alcohols was determined at different temperatures and the activation parameters were calculated at $298 \mathrm{~K}$

TABLE-2

RATE CONSTANTS AND ACTIVATION PARAMETERS FOR THE OXIDATION OF SECONDARY ALCOHOLS BY BTMACB

\begin{tabular}{|c|c|c|c|c|c|c|c|}
\hline \multirow{2}{*}{ Alcohol } & \multicolumn{4}{|c|}{$10^{4} \mathrm{k}_{2}\left(\mathrm{dm}^{3} \mathrm{~mol}^{-1} \mathrm{~s}^{-1}\right)$} & \multirow{2}{*}{$\begin{array}{c}\Delta \mathrm{H}^{*} \\
\left(\mathrm{~kJ} \mathrm{~mol}^{-1}\right)\end{array}$} & \multirow{2}{*}{$\begin{array}{c}-\Delta \mathrm{S}^{*} \\
\left(\mathrm{~J} \mathrm{~mol}^{-1} \mathrm{~K}^{-1}\right)\end{array}$} & \multirow{2}{*}{$\begin{array}{c}\Delta \mathrm{G}^{*} \\
\left(\mathrm{~kJ} \mathrm{~mol}^{-1}\right)\end{array}$} \\
\hline & $288 \mathrm{~K}$ & $298 \mathrm{~K}$ & $308 \mathrm{~K}$ & $318 \mathrm{~K}$ & & & \\
\hline $\mathrm{Me}$ & 16.2 & 35.1 & 75.6 & 153 & $54.6 \pm 0.3$ & $109 \pm 1$ & $87.0 \pm 0.3$ \\
\hline Et & 26.1 & 57.6 & 117 & 225 & $52.1 \pm 0.3$ & $114 \pm 1$ & $85.8 \pm 0.3$ \\
\hline$n$-Pr & 47.7 & 92.7 & 180 & 333 & $46.9 \pm 0.4$ & $127 \pm 1$ & $84.6 \pm 0.3$ \\
\hline$i-\operatorname{Pr}$ & 77.4 & 144 & 279 & 486 & $44.5 \pm 0.6$ & $131 \pm 2$ & $83.5 \pm 0.4$ \\
\hline$i-\mathrm{Bu}$ & 126 & 225 & 387 & 621 & $38.1 \pm 0.3$ & $149 \pm 1$ & $82.4 \pm 0.3$ \\
\hline $\mathrm{ClCH}_{2}$ & 0.31 & 0.80 & 2.03 & 4.68 & $66.6 \pm 0.4$ & $100 \pm 1$ & $96.4 \pm 0.3$ \\
\hline $\mathrm{MeOCH}_{2}$ & 2.52 & 5.94 & 13.5 & 29.7 & $60.1 \pm 0.3$ & $106 \pm 1$ & $91.4 \pm 0.2$ \\
\hline $\mathrm{Ph}$ & 110 & 207 & 405 & 783 & $47.5 \pm 0.7$ & $118 \pm 2$ & $82.6 \pm 0.6$ \\
\hline Benzhydrol & 117 & 225 & 432 & 837 & $47.4 \pm 0.7$ & $118 \pm 3$ & $82.4 \pm 0.6$ \\
\hline Benzhydrol- $\alpha-D$ & 19.8 & 40.1 & 79.2 & 161 & $50.5 \pm 0.8$ & $112 \pm 3$ & $86.7 \pm 0.6$ \\
\hline $\mathrm{k}_{\mathrm{H}} / \mathrm{k}_{\mathrm{D}}$ & 5.91 & 5.61 & 5.45 & 5.20 & - & - & - \\
\hline
\end{tabular}




\begin{tabular}{|c|c|c|c|c|c|c|}
\hline \multicolumn{7}{|c|}{$\begin{array}{c}\text { TABLE-3 } \\
\text { EFFECT OF BENZYLTRIMETHYLAMMONIUM CHLORIDE OR POTASSIUM } \\
\text { BROMIDE ON THE RATE OF OXIDATION OF 2-PROPANOL BY BTMACB }\end{array}$} \\
\hline$[\mathrm{BTMACB}]=0.001 \mathrm{~mol} \mathrm{dm}^{-3}$ & \multicolumn{2}{|c|}{ [Alcohol] $=1 \mathrm{~mol}\left(\mathrm{dm}^{-3}\right)$} & \multicolumn{4}{|c|}{ Temperature $=298 \mathrm{~K}$} \\
\hline $10^{3}[\mathrm{BTMACl}]$ or $[\mathrm{KBr}]\left(\mathrm{mol} \mathrm{dm}^{-3}\right)$ & 0.00 & 0.5 & 1.0 & 2.0 & 3.0 & 4.0 \\
\hline \multicolumn{7}{|l|}{$10^{4} \mathrm{k}_{\text {obs }}\left(\mathrm{s}^{-1}\right)$} \\
\hline BTMACl & 35.1 & 32.4 & 33.3 & 36.0 & 34.2 & 35.0 \\
\hline $\mathrm{KBr}$ & 35.1 & 36.1 & 34.0 & 33.1 & 35.2 & 34.5 \\
\hline \multicolumn{7}{|c|}{ TABLE-4 } \\
\hline$[$ BTMACB $]=0.001 \mathrm{~mol} \mathrm{dm}^{-3}$ & \multicolumn{4}{|c|}{$[2$-Propanol $]=1.00 \mathrm{~mol} \mathrm{dm}^{-3}$} & \multicolumn{2}{|c|}{ Temperature $=298 \mathrm{~K}$} \\
\hline $\mathrm{AcOH} \%$ & 25 & 40 & 50 & & 60 & 72 \\
\hline $10^{4} \mathrm{k}_{2}\left(\mathrm{~s}^{-1}\right)$ & 180 & 68.4 & 35.1 & & 17.1 & 6.30 \\
\hline
\end{tabular}

(Table-2). A plot of $\log \mathrm{k}_{2}$ at different temperature is linearly related to the inverse of the absolute temperature (Fig. 2). The Arrhenius equation is, therefore, valid.

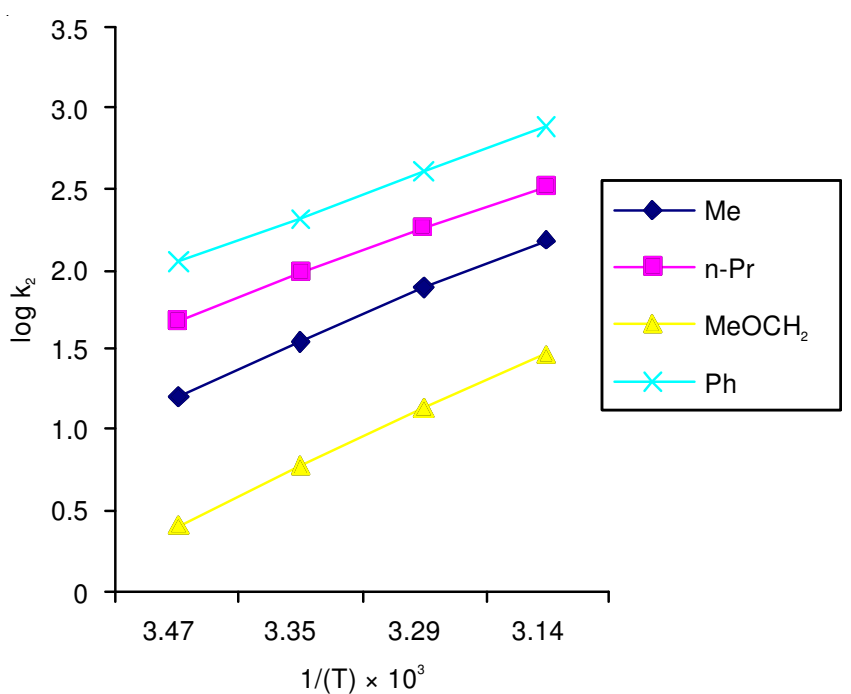

Fig. 2. Oxidation of alcohols by BTMACB: Effect of temperatures

There is a fair correlation between the activation enthalpies and entropies of the oxidation of eight alcohols $\left(r^{2}=0.9281\right)$, indicating the operation of a compensation effect ${ }^{14}$. A correlation between the calculated values of enthalpies and entropies is often vitiated by the experimental errors associated with them. Exner ${ }^{15}$ has suggested an alternative method of testing the validity of the isokinetic relationship. An Exner's plot between $\log \mathrm{k}_{2}$ at 288 and $318 \mathrm{~K}$ was linear $\left(\mathrm{r}^{2}=0.9966\right.$; slope $0.8272 \pm 0.0198$ ) (Fig. 3). The value of isokinetic temperature evaluated from this plot is $650 \pm 94 \mathrm{~K}$. The linear isokinetic correlation suggests that all the alcohols are oxidized by the same mechanism and the changes in the rates are governed by the changes in both the enthalpy and entropy of the activation.

Reactive oxidizing species: Some conductivity measurements have been carried out to determine the nature of BTMACB in aqueous acetic acid solution ${ }^{16}$. It was observed that acetic acid has very low conductivity. Addition of BTMACB increases the conductivity of acetic acid. We measured the conductivity of BTMACB in solvents containing different proportions of acetic acid (100-30\%) and water also. We found that the conductivity increases sharply as the water content is initially increased but reaches a limiting value in about $60 \%$

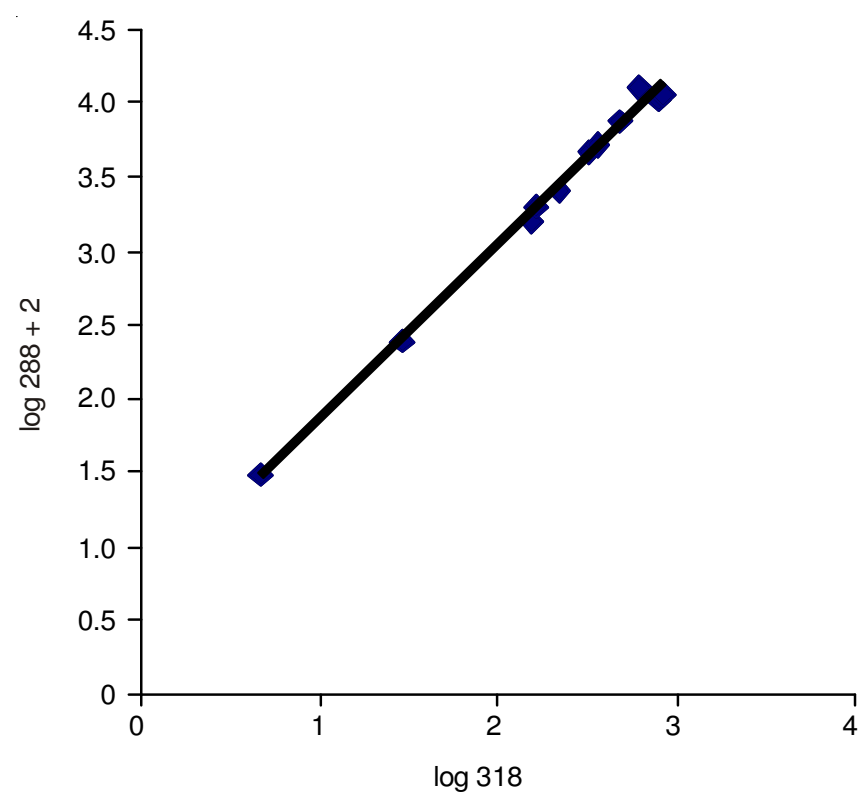

Fig. 3. Exner's isokinetic relationship in the oxidation of alcohols by BTMACB

acetic acid-water mixture. Therefore, BTMACB can be considered as an ionic compound, which exists under our reaction conditions as benzyltrimethylammonium and chlorobromate ions [eqn.(2)]. No effect of added benzyltrimethylammonium ion also indicates that the equilibrium (2) lies far towards the right.

$\mathrm{PhCH}_{2} \mathrm{Me}_{3} \mathrm{NBr}_{2} \mathrm{Cl} \rightleftharpoons \mathrm{PhCH}_{2} \mathrm{Me}_{3} \mathrm{~N}^{+}+\mathrm{Br}_{2} \mathrm{Cl}$

The following equilibria may also exist in the solution.

$$
\begin{aligned}
\mathrm{Br}_{2} \mathrm{Cl}^{-} & \rightleftharpoons \mathrm{Br}_{2}+\mathrm{Cl}^{-} \\
\mathrm{Br}_{2}+\mathrm{H}_{2} \mathrm{O} & \rightleftharpoons \mathrm{HOBr}+\mathrm{HBr}
\end{aligned}
$$

The probable oxidizing species in a solution of BTMACB are, therefore, chlorobromate ion, molecular bromine or hypobromous acid. The equilibria (3) and (4) are likely to be suppressed by the addition of BTMACl or potassium bromide. The absence of any added $\mathrm{BTMACl}$ and bromide ion on the reaction rate rules out role of $\mathrm{Br}_{2}$ and $\mathrm{HOBr}$ in the oxidation process. The oxidation of benzyl alcohol by bromine, in 1:1(v/v) acetic acid-water, is retarded by the addition of bromide ions ${ }^{17}$ whereas the oxidation by BTMACB is unaffected by bromide ions. This indicates that in the present reaction bromine is not the active oxidizing species. Similar results were obtained in the oxidation of aliphatic aldehydes by BTMACB ${ }^{16}$. Thus in 
TABLE-5

TEMPERATURE DEPENDENCE OF THE REACTION CONSTANTS

\begin{tabular}{cccccc}
\hline Temperature $(\mathrm{K})$ & $\rho^{*}$ & $\delta$ & $\mathrm{R}^{2}$ & sd & $\Psi$ \\
\hline 288 & $-1.80 \pm 0.01$ & $-0.73 \pm 0.01$ & 0.9999 & 0.009 & 0.01 \\
298 & $-1.71 \pm 0.01$ & $-0.64 \pm 0.01$ & 0.9998 & 0.010 & 0.02 \\
308 & $-1.62 \pm 0.02$ & $-0.55 \pm 0.02$ & 0.9999 & 0.008 & 0.01 \\
318 & $-1.54 \pm 0.01$ & $-0.46 \pm 0.01$ & 0.9989 & 0.007 & 0.04 \\
\hline
\end{tabular}

the present reaction also the reactive oxidizing species is the chlorobromate ion.

Solvent composition effect: The increase in the rate constant with an increase in the polarity of the medium suggests that the transition state is more polar than the reactants. The plot of $\log \mathrm{k}_{2}$ against the inverse of the dielectric constant is nonlinear. The solvent effect was analysed using GrunwaldWinstein equation ${ }^{18}$.

$$
\log \mathrm{k}_{2}=\log \mathrm{k}_{0}+m \mathrm{Y}
$$

The plot of $\log \mathrm{k}_{2}$ versus $\mathrm{Y}$ is linear $\left(\mathrm{r}^{2}=0.9998\right)$ with $m$ $=0.78 \pm 0.01$. The value of $m$ suggests a transition state, which is more polar than the reactants. Thus considerable charge separation takes place in the transition state of the reaction.

Correlation analysis of reactivity: The rate constants, $\mathrm{k}_{2}$, of oxidation of alcohols failed to yield any significant correlation separately with Taft's ${ }^{19} \sigma^{*}$ and $\mathrm{E}_{\mathrm{s}}$. The correlation with $\sigma^{*}$ accounts for $94 \%$ of the data but correlation is not acceptable by Exner's criterian ${ }^{20}$.

$$
\begin{aligned}
& \log \mathrm{k}_{2}=-1.83( \pm 0.16) \sum \sigma^{*}-2.23 \\
& \mathrm{r}^{2}=0.9540 ; \mathrm{sd}=0.19 ; \mathrm{n}=8 ; \psi=0.23 ; \mathrm{T}=298 \mathrm{~K} \\
& \log \mathrm{k}_{2}=-1.38( \pm 1.02) \sum \mathrm{E}_{\mathrm{s}}-2.90 \\
& \mathrm{r}^{2}=0.2343 ; \mathrm{sd}=0.78 ; \mathrm{n}=8 ; \psi=0.94 ; \mathrm{T}=298 \mathrm{~K}
\end{aligned}
$$

Here $n$ is the number of data points and $\psi$ is the Exner's statistical parameter ${ }^{20}$. The rate constants were, therefore, correlated in terms of Pavelich-Taft's ${ }^{21}$ dual substituent-parameter eqn (8).

$$
\log \mathrm{k}=\rho^{*} \sum \sigma^{*}+\delta \sum \mathrm{E}_{\mathrm{s}}+\log \mathrm{k}_{\mathrm{o}}
$$

The values of the substituent constants were obtained from the compilation by Wiberg ${ }^{19}$. The correlations are excellent; reaction constants being negative (Table-5). There is no significant collinearity $\left(r^{2}=0.2136\right)$ between $\sigma^{*}$ and $E_{s}$ of the nine substituents.

The negative polar reaction constant indicates an electrondeficient carbon centre in the transition state of the rate-determining step. The negative steric reaction constant, points to a steric acceleration of the reaction. This may be explained on the basis of the high ground state energy of the sterically crowded alcohols. Since the crowding is relieved in the product, acetone as well as in the transition state leading to it, the transition state energy of the crowded and uncrowded alcohols do not differ much and steric acceleration, therefore, results. The faster oxidation of 1-phenylethanol and benzhydrol may well be due to the stabilization of the electron-deficient carbon centre in he transition state by phenyl groups through resonance.

Mechanism: There is no kinetic evidence for the formation of an intermediate complex in the present reaction. However, its formation in small amounts cannot be ruled out. It is possible that in the present reaction also an intermediate complex is formed in a rapid pre-equilibrium but the equilibrium constant is very small. The presence of a substantial primary kinetic isotope effect $\left(\mathrm{k}_{\mathrm{H}} / \mathrm{k}_{\mathrm{D}}=5.61\right.$ at $\left.298 \mathrm{~K}\right)$ confirms that the $\alpha-\mathrm{C}-\mathrm{H}$ bond is cleaved in the rate-determining step. In view of any effect of radical scavenger, acrylonitrile, on the reaction rate, it is unlikely that a one-electron oxidation giving rise to free radical is operative in this reaction. However, with the present

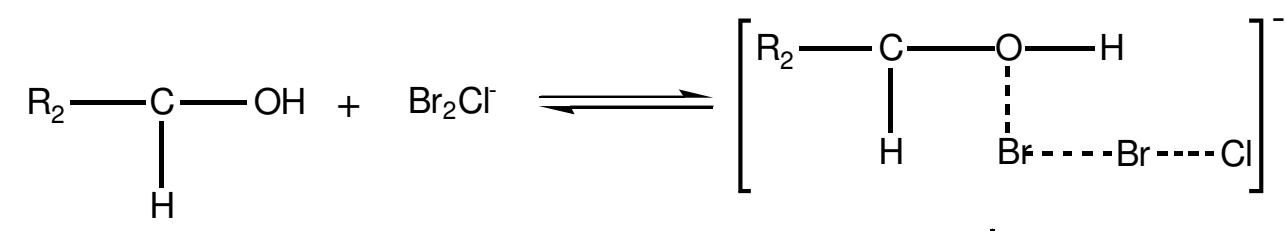

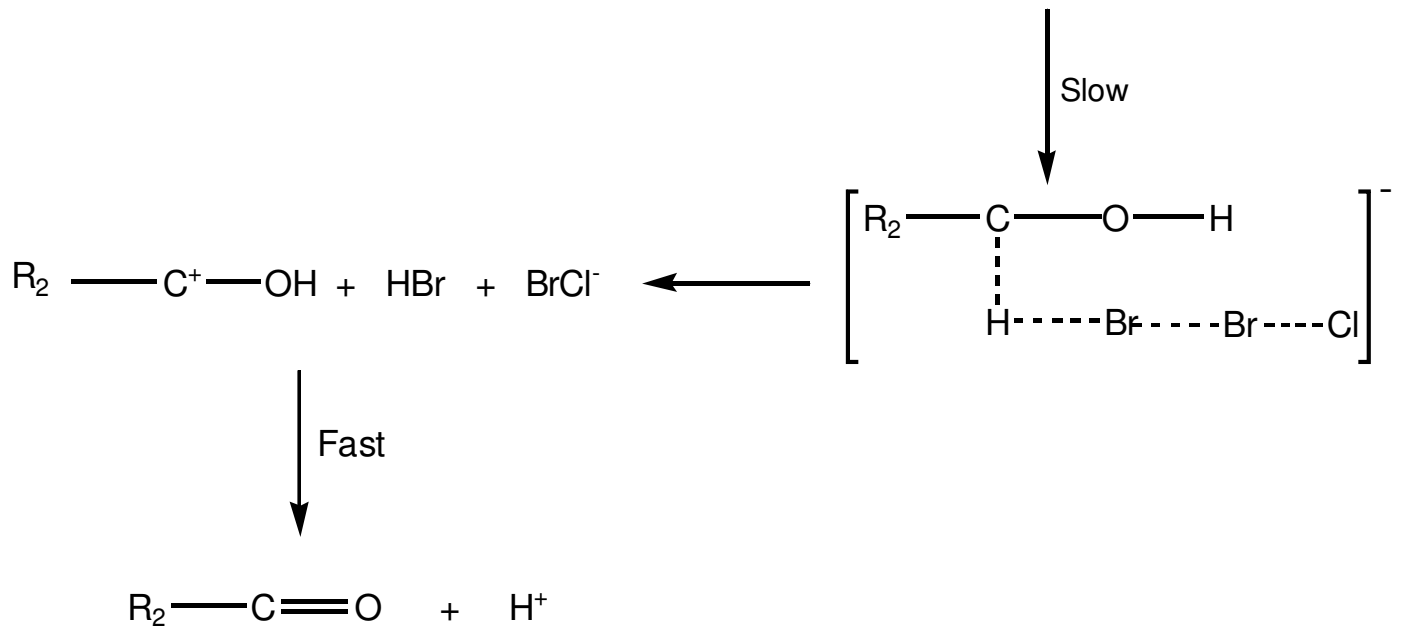


data, it is not possible to state definitely about the nature of the complex. Heasley and co-workers ${ }^{22}$ have postulated the formation of an intermediate $\pi$-complex in the reaction of alkenes with pyridinium hydrobromide perbromide. The formation of similar complexes has also been postulated in the oxidation of aldehydes ${ }^{23}$ and diols ${ }^{24}$ with hexamethylenetetramine-bromine as well as in the oxidation of alcohols ${ }^{25}$ and organic acids ${ }^{26}$ by pyridinium hydrobromide perbromide. The formation of moderately stable complexes is supported by the values of thermodynamic parameters also. The complex formation is favoured by the enthalpy term but there is a loss of entropy indicating the formation of a rigid structure.

The large negative reaction constant and a substantial deuterium kinetic isotope effect suggest a considerable carbocation character in the transition state. Therefore, the following mechanism (Scheme-I) involving transfer of a hydride ion from the alcohol to the oxidant, in the rate-determining step, is suggested.

The observed negative entropy of activation also supports it. As the charge separation takes place, the charged ends become highly solvated. This results in an immobilization of a large number of solvent molecules, reflected in the loss of entropy ${ }^{27}$.

\section{ACKNOWLEDGEMENTS}

Thanks are due to UGC, New Delhi for financial support in the form of BSR-One Time Grant No. F. 4 - 10/2010 (BSR) dated 07.03.2012.

\section{REFERENCES}

1. S. Kajigaeshi, T. Kakinami, M. Moriwaki, S. Fujisaki and T. Okamoto, Tech. Rep. Yamaguchi Univ., 4, 65 (1987).

2. S. Kajigaeshi, T. Kakinami, H. Yamasaki, S. Fujisaki and T. Okamoto, Bull. Chem. Soc. Jpn., 61, 2681 (1988).
3. S. Kajigaeshi, T. Kakinami, M. Shimizu, M. Takahashi, S. Fujisaki and T. Okamoto, Tech. Rep. Yamaguchi Univ., 4, 139 (1988).

4. S. Mitra and K. Sreekumar, Indian J. Chem., 36B, 133 (1997).

5. V.S. Chouhan and V. Sharma, Int. J. Chem. Sci., 1, 341 (2003).

6. V.S. Chouhan and V. Sharma, J. Indian Chem. Soc., 82, 302 (2005).

7. V.S. Chouhan, M. Sharma and V. Sharma, J. Indian Chem. Soc., 84, 582 (2007)

8. V. Tiwari, A. Meena, A. Daiya, P.T.S.R.K. Prasadrao and V. Sharma, Proc. React. Kinet. Mech., 37, 263 (2012).

9. M. Baghmar and P.K. Sharma, Int. J. Chem. Kinet., 33, 390 (2001).

10. J. Gosain and P.K. Sharma, J. Indian Chem. Soc., 79, 815 (2002); Proc. Indian Acad. Sci. Chem. Sci., 115, 135 (2003).

11. P.K. Sharma, J. Indian Chem. Soc., 81, 291 (2004).

12. N.S. Srinivasan and N. Venkatasubramanian, Tetrahedron, 30, 419 (1974).

13. D.D. Perrin, W.L. Armarego and D.R. Perrin, Purification of Organic Compounds, Pergamon; Oxford (1966).

14. L. Liu and Q.-X. Guo, Chem. Rev., 101, 673 (2001).

15. O. Exner, Collect. Czech. Chem. Commun., 29, 1094 (1964).

16. V.S.R. Raju, P.K. Sharma and K.K. Banerji, Indian J. Chem., 39A, 650 (2000).

17. P. Aukett and I.R.L. Barker, J. Chem. Soc., Perkin Trans. 2, 568 (1972).

18. A.H. Fainberg and S. Winstein, J. Am. Chem. Soc., 78, 2770 (1956).

19. K.B. Wiberg, Physical Organic Chemistry, Wiley, New York; p. 414 (1963).

20. O. Exner, Prog. Phys. Org. Chem., 10, 411 (1973).

21. W.A. Pavelich and R.W. Taft Jr., J. Am. Chem. Soc., 79, 4935 (1957).

22. V.L. Heasley, T.J. Louie, D.K. Luttrul, M.D. Miller, H.B. Moore, D.F. Nogales, A.M. Sauerbrey, A.B. Shevel, T.Y. Shibuya, M.S. Stanley, D.F. Shellhamer and G.E. Heasely, J. Org. Chem., 53, 2199 (1988).

23. A. Pareek, S. Varshney and K.K. Banerji, React. Kinet. Catal. Lett., 60, 127 (1997).

24. H. Gangwani, P.K. Sharma and K.K. Banerji, J. Chem. Res. (S), 180 (1999).

25. D. Mathur, P.K. Sharma and K.K. Banerji, J. Chem. Soc., Perkin Trans. II, 205 (1993).

26. D. Suri, S. Kothari and K.K. Banerji, J. Chem. Res., (S) 228, (M) 1301 (1996).

27. E.S. Gould, Mechanism and Structure in Organic Chemistry, Holt, Rinehart and Winston Inc., New York (1964). 